\title{
SMOOTHING CURVES IN $P^{3}$ WITH $p_{a}=1$
}

\author{
CARMEN A. SÁNCHEZ
}

\begin{abstract}
In [3] Tannenbaum proved that every connected, reduced curve in $P^{3}$ of arithmetic genus 0 may be smoothed. Here we prove, using results of Hartshorne and Hirschowitz [1], that every connected, reduced curve in $P^{3}$ of arithmetic genus 1 is also smoothable.
\end{abstract}

Introduction. Let $X$ be a connected, reduced curve in $P^{3}$. We say that $X$ is smoothable if there exists a flat family of curves $X_{t}$ in $P^{3}$, whose general member $X_{t}$ is smooth and whose special member $X_{0}$ is $X$. In $\S 2$, we prove that a connected, reduced curve in $P^{3}$ of arithmetic genus 1 is smoothable. The proof is based on a result of Hartshorne and Hirschowitz [1] and a formula for the arithmetic genus of a curve given in [2] (see $\S 1$ for its statement). I wish to thank Dr. S. Xambó for many valuable suggestions.

Notations. (i) All schemes will be projective algebraic defined over a fixed algebraically closed field $k$.

(ii) Curve will mean a 1-dimensional scheme.

(iii) For $X$ a scheme of dimension $n$, its arithmetic genus is $p_{a}(X)=1-\chi\left(O_{X}\right)$, where

$$
\chi\left(O_{X}\right)=\sum_{i=0}^{n}(-1)^{i} h^{i}\left(X, O_{X}\right) .
$$

(iv) Given an irreducible curve $X, \pi(X)$ will denote the effective genus, i.e., the arithmetic genus of a nonsingular curve which is birationally equivalent to it; in general, for a reducible curve it is defined as the sum of the effective genera of the irreducible components of the curve.

(v) If $X$ is a curve and $P$ a point of $X$, the order of singularity of $X$ in $P$ is

$$
\delta(P: X)=\operatorname{dim}_{k}\left(\tilde{O}_{X, P} / O_{X, P}\right)
$$

where $\tilde{O}_{X, P}$ denotes the integral closure of the local ring of $X$ at $P$ in its total ring of quotients.

(vi) If $C$ and $D$ are two curves in $P^{3}$ which have no common components through the point $P, i(P ; C \cdot D)$ is the length of $O_{C \cap D}$, where $C \cap D$ is the intersection scheme of $C$ and $D$.

(vii) $P^{3}=P_{k}^{3}=\operatorname{Proj} k\left[x_{0}, \ldots, x_{3}\right]$.

\section{1.}

Proposition $1.1[1,4.5]$. Let $X=C \cup D$ be the union of two nonsingular curves $C, D$ in $P^{3}$ with $H^{1}\left(\mathcal{N}_{C}\right)=H^{1}\left(\mathcal{N}_{D}\right)=0$, meeting quasi-transversally in $\leq 4$

Received by the editors February 3, 1986.

1980 Mathematics Subject Classification (1985 Revision). Primary $14 \mathrm{H} 10$. 
points in general position (i.e., no 3 collinear, no 4 coplanar). Then $H^{1}\left(\mathcal{N}_{X}\right)=0$ and $X$ is smoothable.

Proposition 1.2 [2, TheOREM 2]. For a reduced curve $X$ in $P^{3}$, we have

$$
p_{a}(X)=\pi(X)+\sum_{P \in X} \delta(P: X)-(r-1),
$$

where $r$ is the number of irreducible components of $X$.

REMARK 1.3 [2, Proposition 4]. Let $X_{1}, X_{2}, \ldots, X_{r}(r \geq 2)$ be curves in $P^{3}$, no two of which have common components through a common point $P$, and set $X=\bigcup X_{i}$. Then

$$
\delta(P: X)=\sum_{i=1}^{r} \delta\left(P: X_{i}\right)+\sum_{q=2}^{r} i\left(P ;\left(\bigcup_{i=1}^{q-1} X_{i}\right) \cdot X_{q}\right) .
$$

2 .

THEOREM 2.1. Let $X$ be a connected reduced curve in $P^{3}$ of arithmetic genus 1. Then $X$ is smoothable.

Proof. Let $X_{1}, \ldots, X_{r}$ be the irreducible components of $X$ arranged so that $X_{j} \cap\left(X_{1} \cup \cdots \cup X_{j-1}\right) \neq \varnothing$ for all $j=2, \ldots, r$ (we can suppose this because $X$ is connected). As the intersection points of two components are singular points, we have $\sum_{P \in X} \delta(P: X) \geq r-1$. From this and the fact $\pi(X) \geq 0$, and taking into account the expression of the arithmetic genus of $X(1.2)$, we conclude that if $p_{a}=1$ the only possibilities are the following:

(i) $\sum_{P \in X} \delta(P: X)=r-1$ and $\pi(X)=1$,

(ii) $\sum_{P \in X} \delta(P: X)=r$ and $\pi(X)=0$.

In the first case each component intersects the union of the previous ones at only one point and at most two components meet at any point. The only singular points are the intersection of two components and the order of the singularity in each of them is 1 (i.e., the intersection is quasi-transversal). $\pi(X)=1$ implies that one of the components has genus 1 and the others are rational.

In case (ii), all the components are rational and we have the following cases:

(a) $X_{j} \cap\left(X_{1} \cup \cdots \cup X_{j-1}\right)=\left\{P_{j}\right\}, j=2, \ldots, r, P_{i} \neq P_{j}$ for $i \neq j$, the intersection being quasi-transversal and one of the components having a singularity of order 1 (node).

(b) $X_{j} \cap\left(X_{1} \cup \cdots \cup X_{j-1}\right)=\left\{P_{j}\right\}, j=2, \ldots, r, j \neq k$, and $X_{k} \cap\left(X_{1} \cup \cdots \cup X_{k-1}\right)=$ $\left\{P_{k}, Q_{k}\right\}, P_{i} \neq P_{j}$ for $i \neq j, P_{i} \neq Q_{k}$ for all $i$, and the intersections being quasitransversal.

(c) $X_{j} \cap\left(X_{1} \cup \cdots \cup X_{j-1}\right)=\left\{P_{j}\right\}, j=2, \ldots, r, P_{i} \neq P_{j}$ for $i \neq j$ except for $i=2, j=3$ (i.e., there are three components meeting at the same point $(\delta=3)$ ) and all the intersections are quasi-transversal.

(d) $X_{j} \cap\left(X_{1} \cup \cdots \cup X_{j-1}\right)=\left\{P_{j}\right\}, j=2, \ldots, r, P_{i} \neq P_{j}$ for $i \neq j$, and all the intersections being quasi-transversal except one of them, which is a tangency with $\delta=2$.

So, $X$ will be of one of the types shown in the figure.

To prove that $X$ is smoothable, we analyze all the possible cases. 


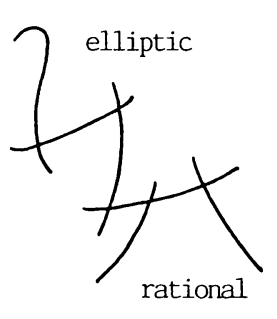

I

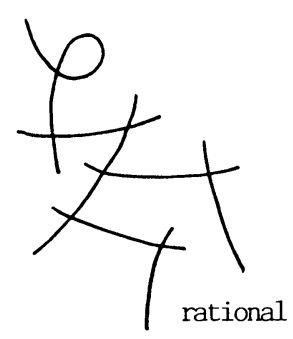

II

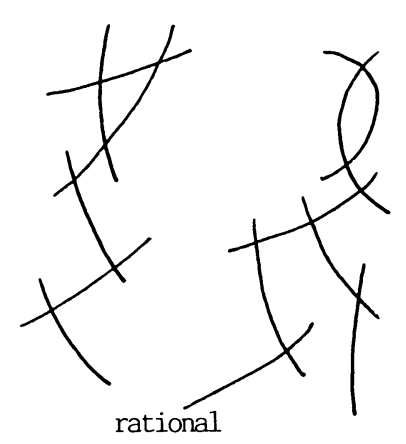

III

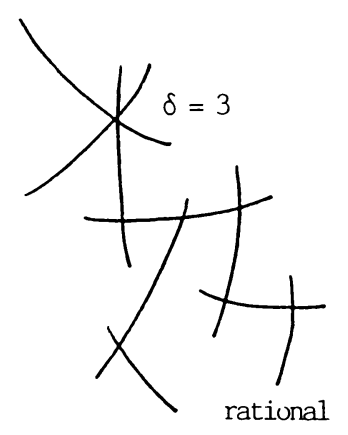

IV

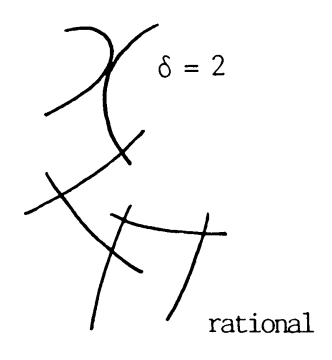

$\mathrm{V}$

Case I. Let $X_{1}$ be the elliptic component. As any divisor of positive degree on an elliptic curve is nonspecial, we have $H^{1}\left(O_{X_{1}}(1)\right)=0$ and so $H^{1}\left(\mathcal{N}_{X_{1}}\right)=0$. Moreover, if $C$ is a rational curve of degree $d, H^{1}\left(O_{C}(1)\right)=H^{1}\left(O_{P^{1}}(d)\right)=0$. Hence, every rational curve is nonspecial and consequently $H^{1}\left(\mathcal{N}_{C}\right)=0$. So we can apply Proposition 1.1 to the curves $X_{1}$ and $X_{2}$, obtaining that $X_{1} \cup X_{2}$ is smoothable. Now taking cohomology in the exact sequence

$$
0 \rightarrow O_{X_{1} \cup X_{2}} \rightarrow O_{X_{1}} \oplus O_{X_{2}} \rightarrow O_{P_{2}} \rightarrow 0
$$

tensored by $O(1)$ and using that $H^{1}\left(O_{X_{i}}(1)\right)=0$ for $i=1,2$ and that the map $H^{0}\left(O_{X_{1}}(1)\right) \oplus H^{0}\left(O_{X_{2}}(1)\right) \rightarrow H^{0}\left(O_{P_{2}}\right)$ is an epimorphism (because $H^{0}\left(O_{X_{2}}(1)\right) \rightarrow$ $H^{0}\left(O_{P_{2}}\right)$ is an epimorphism), we conclude that $H^{1}\left(O_{X_{1} \cup X_{2}}(1)\right)=0$.

Let $X_{t}^{\prime}$ be a family smoothing $X^{\prime}=X_{1} \cup X_{2}$. By semicontinuity, $H^{1}\left(O_{X_{t}^{\prime}}(1)\right)=0$, and so we can add a rational curve $Y_{t}$ meeting $X_{t}^{\prime}$ in one point to get a family $X_{t}^{\prime} \cup Y_{t} \rightarrow X^{\prime} \cup X_{3}$. Now $X_{t}^{\prime}$ is nonsingular and nonspecial, so by (1.1) $X_{t}^{\prime} \cup Y_{t}$ is smoothable and hence $X^{\prime} \cup X_{3}$ is also smoothable. Arguing as before, starting now from the exact sequence

$$
0 \rightarrow O_{X^{\prime} \cup X_{3}} \rightarrow O_{X^{\prime}} \oplus O_{X_{3}} \rightarrow O_{P_{3}} \rightarrow 0,
$$

we obtain that $X^{\prime} \cup X_{3}$ is nonspecial.

Hence we see that when we add a rational component, the new curve is smoothable and nonspecial. Then we can repeat this process until we exhaust all the components, obtaining finally that $X$ is smoothable.

Case II. Let $X_{1}$ be the component with the node. $X_{1}$ is smoothable and nonspecial $[\mathbf{1}, 1.3]$, which allows us to proceed as in the previous case. 
Case III. The argument given for Case I is still valid here since we can apply (1.1) when we add a component meeting the curve at two points.

Case IV. The proof is similar to the proof of Case I because all intersections are quasi-transversal.

Case V. Let $X_{1}$ and $X_{2}$ be the components which are tangent. First let us see that $X_{1} \cup X_{2}$ is smoothable. In fact we will construct a deformation of $X_{1} \cup X_{2}$ to a reducible curve with two rational components meeting at two points. Let $\psi_{1}: P^{1} \rightarrow P^{3}$ and $\psi_{2}: P^{1} \rightarrow P^{3}$ be parametrizations of $X_{1}$ and $X_{2}$, respectively, such that $x_{0}=\psi_{1}(0)=\psi_{2}(0)$ is the point of contact. For all $t$, except possibly a finite number, we can define $\sigma_{t} \in \operatorname{PGL}\left(P^{3}\right)$ depending algebraically on $t$, with $\sigma_{0}=$ Id and such that $\sigma_{t}\left(x_{0}\right)=x_{0}$ and $\sigma_{t}\left(\psi_{1}(t)\right)=\psi_{2}(t)$. As the condition $\#\left(\sigma_{t}\left(X_{1}\right) \cap X_{2}\right) \geq 3$ is closed, there is an open set $U$ in $P^{1}$ with $0 \in U$ and such that $\sigma_{t}\left(X_{1}\right) \cap X_{2}=\left\{x_{0}, \psi_{2}(t)\right\}$. Then $\sigma_{t}\left(X_{1}\right) \cup X_{2}$ will be a flat family (because the degree and arithmetic genus are constant) whose special member is $X_{1} \cup X_{2}$. By (1.1), $\sigma_{t}\left(X_{1}\right) \cup X_{2}$ is smoothable, and hence $X_{1} \cup X_{2}$ is also smoothable. As before, we see that $X_{1} \cup X_{2}$ is nonspecial and now we can proceed as in the previous cases.

REMARK 2.2. We can think of applying similar techniques to study the smoothability of curves with arithmetic genus 2 . In such a case the number of possibilities increases considerably. Most of the cases can be decided as before, but for two cases we still do not know whether they are smoothable or not, namely

(i) the union of two nonsingular curves meeting quasi-transversally at only one point, one of genus 2 and the other rational,

(ii) the union of two nonsingular curves which are tangent at one point, one elliptic and the other rational.

\section{REFERENCES}

1. R. Hartshorne and A. Hirschowitz, Smoothing algebraic space curves, Algebraic Geometry, Proceedings Sitges 1983, Lecture Notes in Math., vol. 1124, Springer-Verlag, Berlin and New York, 1985, pp. 98-131.

2. H. Hironaka, On the arithmetic genera and the effective genera of algebraic curves, Mem. Coll. Sci. Univ. Kyoto 30 (1957), 177-195.

3. A. Tannenbaum, Degenerations of curves in $P^{3}$, Proc. Amer. Math. Soc. 68 (1978), 6-10.

Departamento de Algebra y Geometria, Facultad de Matemáticas, UniVerSidAd de BARCElona, Gran Via de les Corts Catalanes, 585, 08007-BarCelona, SPAIN 